\title{
Replication of the LINGO1 gene association with essential tremor in a North American population
}

\author{
Lorraine N Clark ${ }^{1,2,3}$, Naeun Park ${ }^{4}$, Sergey Kisselev ${ }^{2}$, Eileen Rios ${ }^{4}$, Joseph H Lee ${ }^{1,3,4,5}$ and Elan D Louis ${ }^{1,4,5,6}$
}

A marker in the LINGO1 gene, rs9652490, showing significant genome-wide association with essential tremor (ET), was recently reported in an Icelandic population. To replicate this association in an independent population from North America, we genotyped 15 SNPs in the LINGO1 gene in 257 Caucasian ET cases ('definite,' 'probable' or 'possible') and 265 controls enrolled in an epidemiological study at Columbia University. We observed a marginally significant association with allele $G$ of the marker rs9652490 $(P=0.0569$, odds ratio $(O R)=1.33)$. However, for 'definite' or 'probable' $E T$, rs 9652490 was significantly associated with ET $(P=0.03,0 R=1.41)$. Our subsequent analysis of early-onset $\mathrm{ET}$ (age at onset $<40$ years) revealed that three SNPs, rs 177008 , rs 13313467 and rs8028808, were significantly associated with $\mathrm{ET}(P=0.028,0 R=1.52 ; P=0.0238$, $\mathrm{OR}=1.54$; and $P=0.0391, \mathrm{OR}=1.55$, respectively). These three $\mathrm{SNPs}$ represent a $2.3 \mathrm{~kb}$ haplotype. Finally, a meta-analysis of three published studies confirms allelic association with rs9652490 and two adjacent SNPs. Our study independently confirms that the LINGO1 gene is a risk factor for ET in a Caucasian population in North America, and further shows that those with early-onset ET are likely to be at high risk.

European Journal of Human Genetics (2010) 18, 838-843; doi:10.1038/ejhg.2010.27; published online 7 April 2010

Keywords: essential tremor; LINGO1; association; risk factor

\section{INTRODUCTION}

Essential tremor (ET) is one of the most common neurological diseases, ${ }^{1}$ with a prevalence (age $\geq 40$ years) estimated to be $4.0 \%$ and prevalence in the oldest old exceeding $20.0 \% .^{2}$ The underlying etiological factors and disease mechanisms are not well understood and little is known about the contribution of genetic risk factors to ET. Linkage studies in families with ET have identified three gene loci, namely, ETM1 (OMIM 190300), ETM2 (OMIM 602134) and ETM3 (OMIM 611456); $;^{3-5}$ however, the causal genes have yet to be identified and candidate gene studies in ET case-control populations have also failed to replicate associations. Several clinical and post-mortem studies suggest an association between ET and Parkinson's disease $(\mathrm{PD})^{6-8}$ and studies have investigated whether genetic risk factors for PD may also contribute to the genetic etiology of ET. Our own and other published studies suggest that two of the most significant risk factors for PD, LRRK2 and GBA, do not contribute to ET ${ }^{9-13}$ Recently, a genome-wide association study of ET in an Icelandic population identified a marker in the LINGO1 gene to be significantly associated $\left(P=1.2 \times 10^{-9}\right.$; odds ratio $\left.(\mathrm{OR})=1.55\right)$, which was replicated in the same report in follow-up samples from Austria $(P=0.0082 ; \mathrm{OR}=1.73)$, Germany $(P=0.15 ; \mathrm{OR}=1.39)$ and the United States $(P=0.14$; $\mathrm{OR}=1.32) .{ }^{14}$

In this study, we further examined the LINGO1 gene using detailed clinical and ethnic background information obtained from a casecontrol (257 ET cases vs 265 controls) study conducted in northern
Manhattan. The study had four aims. First, using our sample, we performed a case-control association analysis to evaluate the association of the marker rs9652490 and further characterized its relation with clinical subtype. Second, we performed a haplotype analysis using seven SNPs flanking rs9652490. Third, we performed a metaanalysis of three published studies ${ }^{14-16}$ in addition to our own study. Finally, we evaluated the frequency in our sample of seven variants that were previously identified through sequencing of LINGO1 exons in Icelandic ET cases; these variants included four 'synonymous' coding SNPs, a variant located in the $5^{\prime}$ UTR, and two SNPs located in the $3^{\prime}$ UTR.

\section{MATERIALS AND METHODS}

\section{Subjects}

ET cases and controls were enrolled in an epidemiological study at the Neurological Institute, Columbia University, beginning in 2000. Each signed a written informed consent approved by the University Human Ethics Committee. ET cases were recruited from the Neurological Institute. Controls, ascertained from the same set of zip codes in New York, New Jersey and Connecticut as cases, were recruited using random-digit telephone dialing and were frequency-matched on age (5-year strata), gender and race categories. Each control was initially screened for tremor using a questionnaire and later underwent the same detailed videotaped neurological examination and tremor examination as the cases to ensure that they did not have ET. All participants underwent a demographic and medical history questionnaire, a family history questionnaire (any first- or second-degree relative with tremor (nonspecific),

\footnotetext{
${ }^{1}$ Taub Institute for Research on Alzheimer's Disease and the Aging Brain, College of Physicians and Surgeons, Columbia University, New York, NY, USA; ${ }^{2}$ Department of Pathology and Cell Biology, College of Physicians and Surgeons, Columbia University, New York, NY, USA; ${ }^{3}$ Center for Human Genetics, College of Physicians and Surgeons, Columbia University, New York, NY, USA; ${ }^{4}$ Gertrude H. Sergievsky Center, College of Physicians and Surgeons, Columbia University, New York, NY, USA; ${ }^{5}$ Department of Epidemiology, Mailman School of Public Health, Columbia University, New York, NY, USA; 'Department of Neurology, College of Physicians and Surgeons, Columbia University, New York, NY, USA

Correspondence: Professor LN Clark, Taub Institute, Columbia University Medical Center, College of Physicians and Surgeons, 12-420, 630 168th street, New York, NY 10032, USA. Tel: +212 304 5268; Fax: +212 305 5498; E-mail: Ic654@columbia.edu
}

Received 21 July 2009; revised 4 December 2009; accepted 8 January 2010; published online 7 April 2010 
ET or PD), and a videotaped neurological examination and tremor examination. Self-reported information on race and ethnic group was obtained. Beginning in 2002, self-reported information on Jewish ancestry was also collected. Data on age of onset of tremor, which we have shown to be reliable, ${ }^{17}$ were by self-report. On the basis of previous data on the distribution of age of onset in ET, early age of onset was designated as $<40$ years of age. ${ }^{18}$

After review of the history and videotaped examinations, the diagnosis of ET was then reconfirmed by a senior neurologist specializing in movement disorders (E.D.L) using published criteria for possible, probable or definite $\mathrm{ET},{ }^{19}$ with the latter two categories requiring tremor of greater severity. The presence of bradykinesia or any other sign of parkinsonism (except isolated rest tremor) was an exclusionary criterion for ET.

There were initially 699 participants, of whom 617 (88.3\%) were nonHispanic White (328 ET cases and 289 controls). We included in these analyses 265 non-Hispanic white controls and 257 of 328 non-Hispanic white cases whose ET diagnoses were reconfirmed and who had an available sample (total $n=522$ ).

\section{Molecular genetic analysis}

SNP genotyping. LINGO1 SNPs reported by Stefansson et al ${ }^{14}$ were genotyped. LINGO1 SNP-marker genotyping was performed using matrix-assisted laser desorption/ionization time of flight mass spectrometry (Sequenom, San Diego, CA, USA). PCR assays and mass extend reactions were designed using mass array assay design software (Sequenom). SNP details, assays, PCR primers and mass extend primers are provided in Supplementary Table 1. PCR assays were performed using Applied Biosystems (Foster City, CA, USA) Geneamp PCR thermocyclers. Extension products were analyzed using the mass array compact mass spectrometer (Bruker Daltonik, Billerica, MA, USA), and spectra were analyzed using Spectro TYPER 2.0 software (Sequenom). All genotyping was performed in duplicate with separate assays; analyses were performed blind to case-control status.

\section{Statistical analysis}

For our association analyses of LINGO1, we studied 522 non-Hispanic whites (257 ET cases and 265 controls). Before association analysis, we assessed SNP markers in controls for deviations from Hardy-Weinberg equilibrium using the HAPLOVIEW program (http://www.broadinstitute.org/mpg/haploview; Haploview, Cambridge, MA, USA). ${ }^{20}$ The $\chi^{2}$-test (or the Fisher's exact test when samples fewer than five) was used to assess genotypic and allelic associations between ET and each of the SNP markers. The HAPLOVIEW program was used to perform single point analysis as well as estimation of linkage disequilibrium (LD) structure and haplotype blocks. Haplotype analyses were performed with HAPLO.STATS v1.1.1 for case-control data using the same sliding window of two to three contiguous SNPs. ${ }^{21}$ To minimize the risk of a false-positive finding from rare haplotypes, we computed empirical $P$-values by generating the null distribution on the basis of 1000 replicates of haplotype analyses.

We performed meta-analysis to assess whether rs 9652490 and flanking SNPs were significantly associated in the three published studies ${ }^{14-16}$ with our study. For this purpose, we estimated meta-analysis of $P$-values from four studies as implemented in METAL (http://www.sph.umich.edu/csg/abecasis/metal/), which estimates a single summary $P$-value across studies with varying ethnicity, phenotype distribution, sex. An overall $Z$-statistic and $P$-value are calculated while taking into account the number of individuals studied in each study. To assess the association independent of the discovery set and to evaluate the overall effect, we performed two meta-analyses: one excluding the original study by Stefansson $e t \mathrm{al}^{14}$ and one combining all studies.

\section{RESULTS}

Clinical characteristics and demographics of genotyped ET cases and controls

For the LINGO1 analyses, 257 cases and 265 controls were similar in years of education (Table 1). A larger proportion of ET cases were male and Ashkenazi Jewish (AJ), and a higher proportion had a family history of ET (Table 1). Fifteen SNPs in the LINGO1 gene, including rs9652490, were genotyped in 257 ET cases and 265 controls. Details
Table 1 Demographic and clinical characteristics of genotyped subjects

\begin{tabular}{lcc}
\hline & $\begin{array}{c}\text { ET Cases } \\
(\mathrm{N}=257)\end{array}$ & $\begin{array}{c}\text { Controls } \\
(\mathrm{N}=265)\end{array}$ \\
\hline ET diagnosis: definite/probable/possible & $75 / 118 / 64$ & 0 \\
$\%$ Male $(n)^{*}$ & $52.1 \%(134)$ & $42.6 \%(113)$ \\
Mean age at tremor onset (years) (SD) & $43.8(22.7)$ & Not applicable \\
Mean years of education (SD) & $15.3(3.8)$ & $15.6(3.4)$ \\
$\%$ With family history of PD $(n)$ & $9.3 \%(24)$ & $6.0 \%(16)$ \\
$\%$ With family history of ET $(n)^{* *}$ & $34.2 \%(88)$ & $1.1 \%(3)$ \\
$\%$ Ashkenazi Jewish ancestry $(n)^{* *}$ & $36.6 \%(94)$ & $23.4 \%(62)$ \\
\hline
\end{tabular}

${ }^{*} P<0.05,{ }^{* *} P<0.001$.

of the SNPs genotyped in this study are included in Table 2a and Supplementary data.

\section{LINGO1 single point association}

Although allele $\mathrm{G}$ of rs9652490, identified by Stefansson et al ${ }^{14}$ in an Icelandic population, was marginally associated with ET $(P=0.0569$, $\mathrm{OR}=1.33,95 \%$ confidence interval $(\mathrm{CI})=0.99-1.80)$ in this data set (Table 2a), we observed that rs7177008 and rs13313467, located 1.3 and $2.3 \mathrm{~kb}$ away from rs9652490, respectively, were significantly associated with ET in the total sample ( $P=0.0473,0.0393$, respectively) (Table $2 \mathrm{a}$ ). We then restricted the analysis to early-onset ( $<40$ years of age) ET ( $n=104$ ET cases) and 255 controls and focused on the haplotype block (See supplementary Figure 1A) that included candidate SNPs to identify a high-risk group. We observed that the magnitude of association strengthened for $\mathrm{rs} 177008(\mathrm{OR}=1.52,95 \%$ CI: $1.04-2.22 ; P=0.028), \mathrm{rs} 13313467$ ( $\mathrm{OR}=1.54,95 \% \mathrm{CI}: 1.06-2.25$; $P=0.0238)$ and $\mathrm{rs} 8028808(\mathrm{OR}=1.55,95 \% \mathrm{CI}: 1.02-2.35 ; P=0.039)$ (Table 2b). When we compared the cases with a family history of ET $v s$ controls, rs 8028808 remained significant $(\mathrm{OR}=1.58,95 \% \mathrm{CI}: 1.02$, 2.45; $P=0.038$ ), but flanking SNPs no longer reached a $P$-value of $<0.05$ (Table 2c).

To assess whether the association was driven by a group of clinically homogeneous cases, we restricted analysis to ET cases that had received a diagnosis of 'definite' or 'probable' ET ( $n=193$ ET cases) and 265 controls, and found the same three SNPs (rs177008, $P=0.0257$; rs13313467, $P=0.0213$; and rs8028808, $P=0.0329$ ) as well as rs9652490 $(P=0.0328)$ to be significantly associated with disease (data not shown). However, we did not extend this restricted analysis to early onset and to family history of ET because of small numbers of cases in these subgroups.

To determine whether the observed allelic associations were the same in Ashkenazi Jews as in the overall Caucasian samples, we restricted the analysis to 94 ET cases and 62 controls who reported AJ ancestry. This analysis revealed that rs9652490 was no longer associated, but an SNP located in the 3'UTR of LINGO1, rs11853396, showed evidence of association $(P=0.0317, \mathrm{OR}=1.65)$ (data not shown).

\section{Linkage disequilibrium and haplotype analysis}

Our 3-mer sliding window haplotype analyses of SNPs 8-15 suggested a risk haplotype of 'GGGGA' with allele 'G' at rs9652490 in LINGO1 for ET (Table 3). As shown in Table 3, the strong and consistent association was observed in early-onset ET, centering around SNPs 10-12 (empirical $P=0.02$ ). Similarly, haplotypic analysis of the cases 
Table 2a Association between ET and SNPs in the LINGO1 gene for all ET cases vs controls

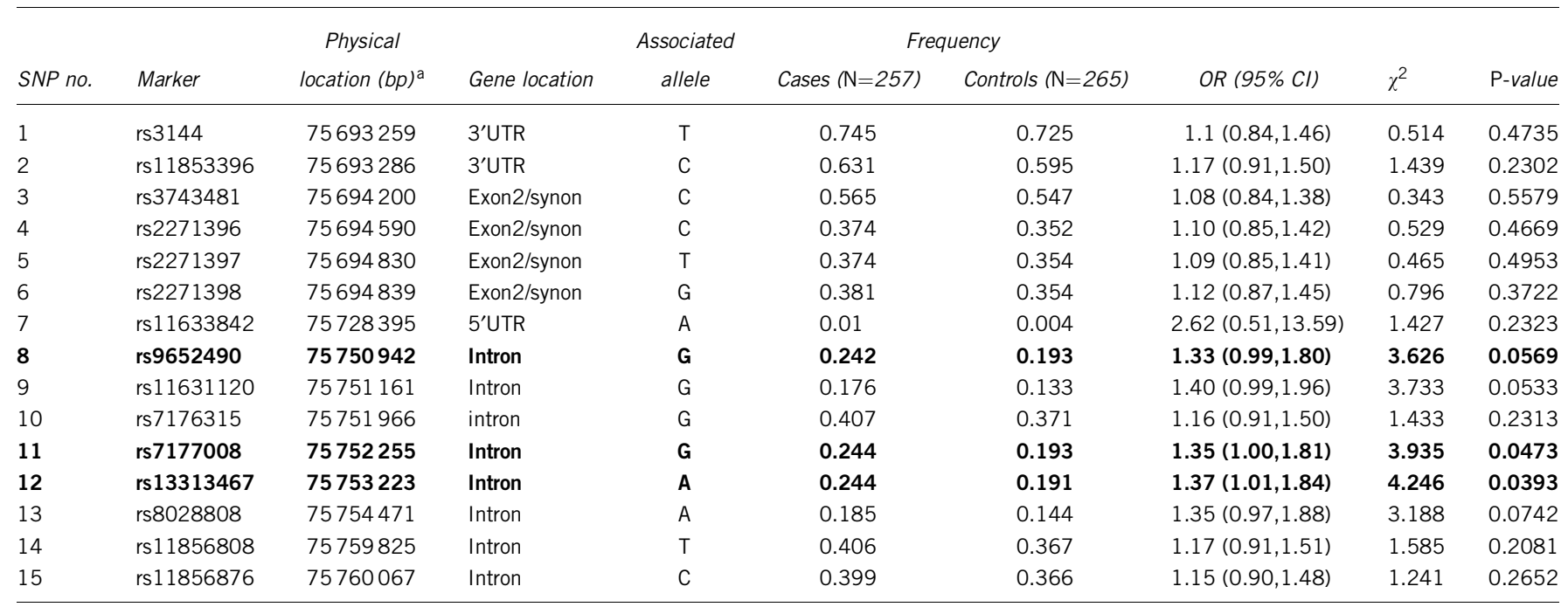

SNPs 1-7 were previously identified by Stefansson et a ${ }^{14}$ by sequencing of the LINGO1 gene in Icelandic ET cases.

SNPs with $P<0.05$ and the SNP identified in the Icelandic study (rs9652490) are in bold.

aGenomic position based on NCBI genome build 36.3.

Table $2 b$ Single point association for early-onset ET cases

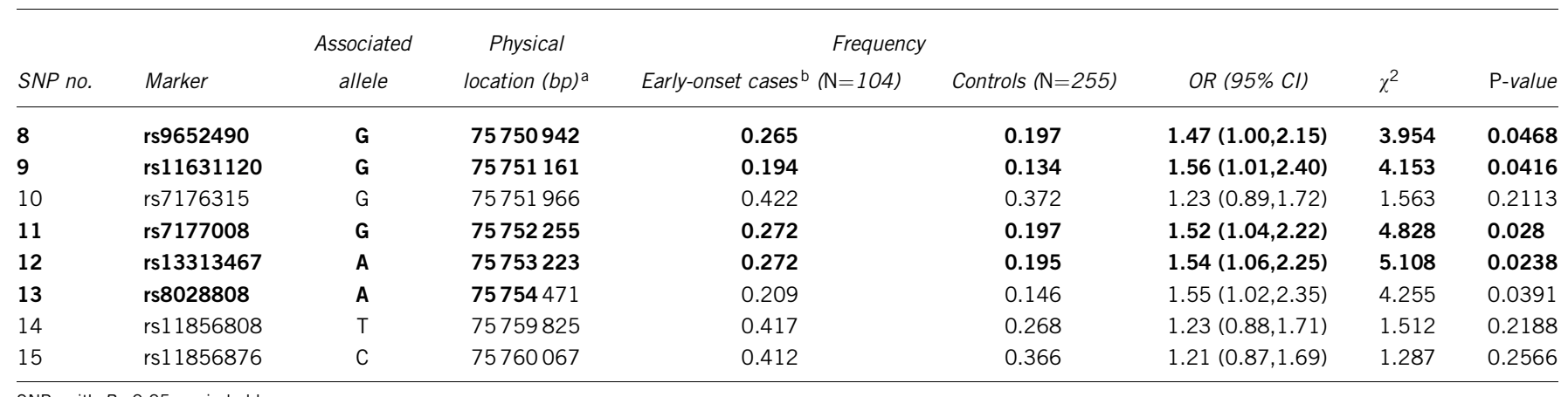

SNPs with $P<0.05$ are in bold.

${ }^{\mathrm{a}}$ Genomic position based on NCBI genome build 36.3 .

bEarly onset defined as those who have definite, probable or possible ET at 40 years of age or younger. For this analysis, we included controls who were $>40$ years of age to serve as 'super' controls.

Table 2c Single point association for ET cases with a family history of ET

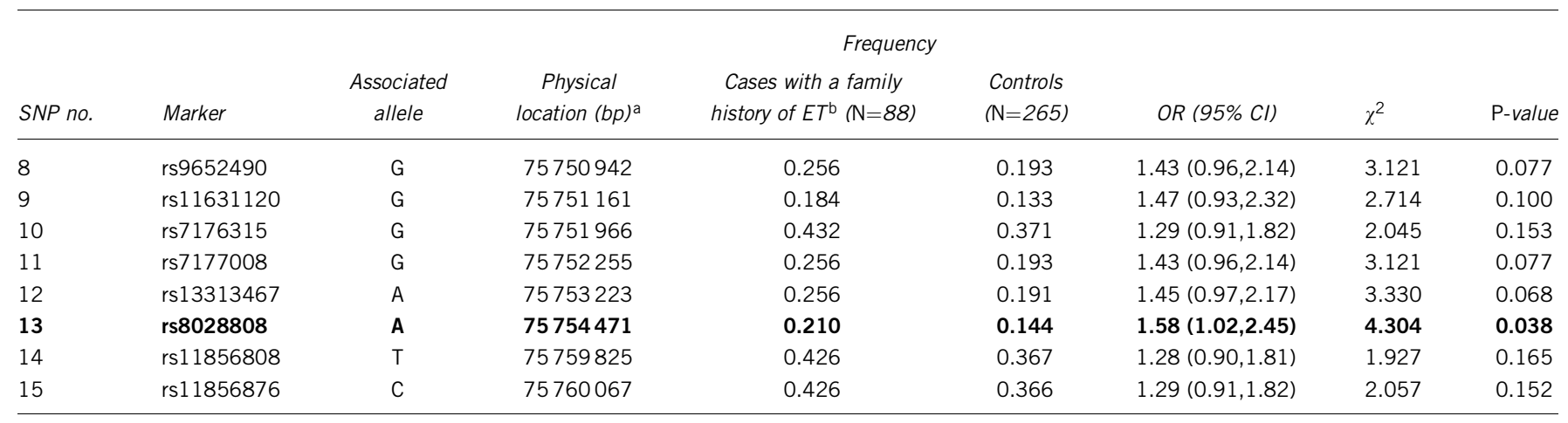

SNPs with $P<0.05$ are in bold.

a Genomic position based on NCBI genome build 36.3.

bSelf-report of a family history in first-degree relatives. 


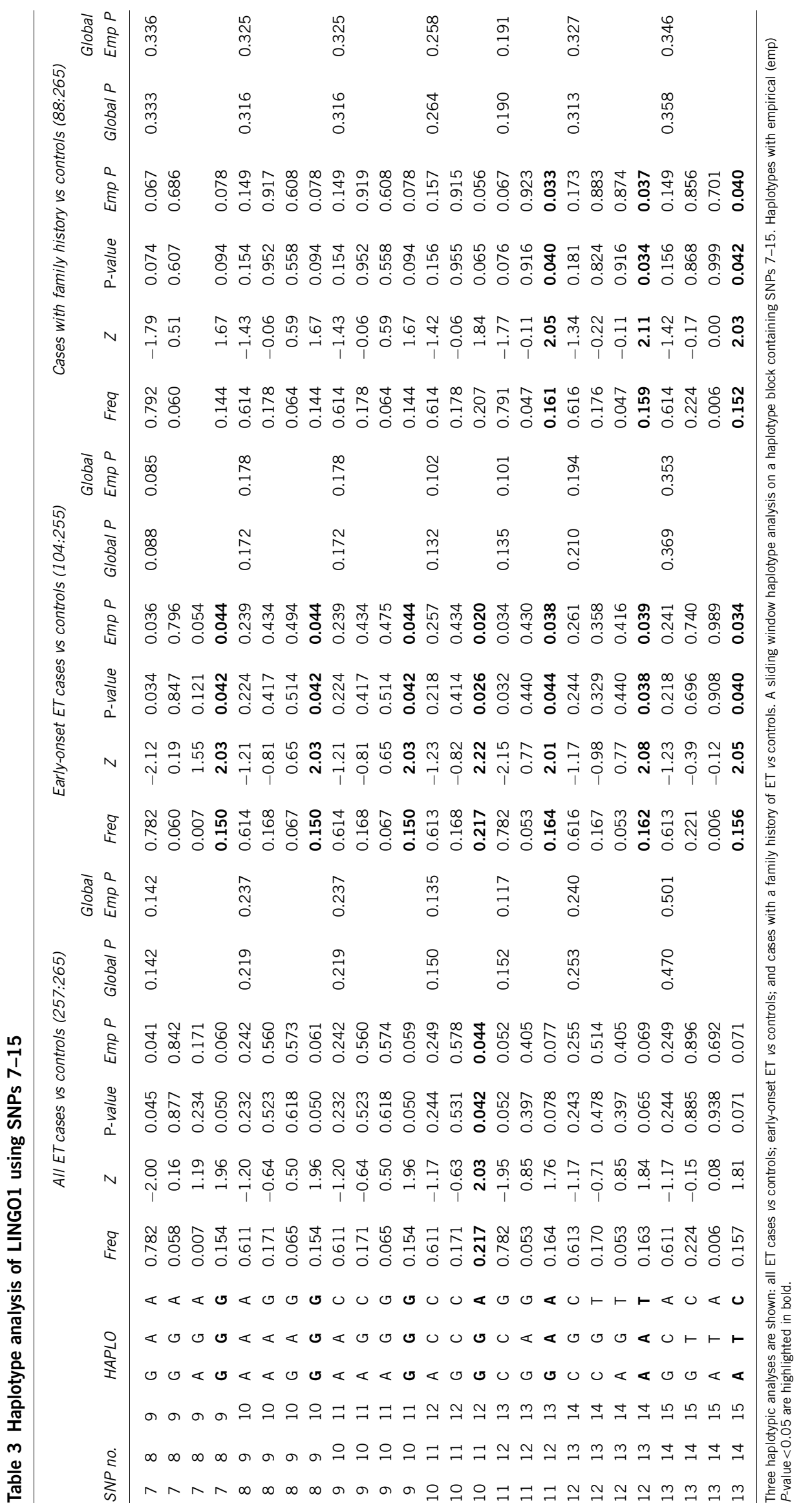


with a family history of ET localized to SNPs 11-15. Among earlyonset ET, this 5-mer haplotype (SNPs 8-12: rs9652490, rs11631120, rs7176315, rs7177008 and rs13313467) spans $2281 \mathrm{bp}$, and was found to be significantly associated with ET in a separate analysis (empirical $P=0.044)$. However, the strength of haplotypic association did not improve when we restricted our analysis to definite and probable ET, suggesting that it is a subset of early-onset ET that is driving the association. The inferred haplotypes for SNPs 7-14 and LD patterns in controls and cases are shown in Table 3 and Supplementary Figure 1A, respectively. LD across these SNPs for Ashkenazi Jewish samples are provided in Supplementary Figure 1A.

\section{Evaluation of LINGO1 sequence variants}

We evaluated the frequency of sequence variants previously identified through sequencing of LINGO1 exons in Icelandic ET cases. No significant association was observed for any of the variants analyzed.

\section{Meta-analysis of SNPs in LINGO1}

The overall meta-analysis confirmed the strong allelic association between rs9652490 and ET $(P=1.55 \mathrm{E}-11)$. Given the large number of subjects $(n=19854)$ studied by Stefansson et $a l,{ }^{14}$ it is not unexpected that the overall observed association remains strong, largely driven by the original finding. Thus, we performed a metaanalysis excluding the subjects from the original study (Table 4). When three replication studies are examined together, the $G$ allele was significantly associated with ET in all three studies $(P=0.0007)$. In addition, the same alleles for the two adjacent SNPs, rs3144 and rs8028808, were associated ( $P=0.02421,0.00148$, respectively) with ET in two of the three studies.

\section{DISCUSSION}

This is one of the first studies to replicate the association of the SNP marker rs9652490 in the LINGO1 gene in an independent population from North America. To confirm the earlier association and to further characterize genotype-phenotype relations, we studied a well-characterized set of ET cases and controls that were enrolled in an epidemiological study at Columbia University and that underwent a demographic and medical history questionnaire, a family history questionnaire (any first- or second-degree relative with tremor (nonspecific), ET or PD) and a videotaped neurological examination and tremor examination. We then performed allelic and haplotypic association based on diagnostic criteria, early-onset ET, family history of ET and AJ ancestry to identify high-risk group(s). Finally, no significant association was observed with sequence variants previously identified through sequencing of LINGO1 exons in Icelandic ET cases.

We extended the Icelandic study to show that SNP rs9652490 was significantly associated with definite or probable ET, and the magnitude of association was strongest for those with early-onset ET. Those who have a family history of ET had the same haplotypes associated with ET, whereas those with AJ ancestry did not demonstrate excess risk. Given the small sample size, further studies are needed. The $2.3 \mathrm{~kb}$ haplotype associated with ET in our study includes the associated SNP, rs9652490, within a $9 \mathrm{~kb}$ block of SNPs in strong LD in intron 3 of LINGO1. We did not observe association of sequence variants previously identified through sequencing of LINGO1 exons in Icelandic ET cases consistent with reported findings. Our overall meta-analysis including three published studies ${ }^{14-16}$ and our own study confirmed the strong allelic association between rs9652490 and ET $(P=1.55 \mathrm{E}-11)$, and the association remained significant $(P=0.0007)$ when we performed a meta-analysis excluding the subjects from the original study from Stefansson et al. ${ }^{14}$ In addition, two adjacent intronic SNPs, rs3144 and rs8028808, were associated with ET in two of the three studies $(P=0.02421,0.00148$, respectively). Thus, the most likely explanation is that rs9652490 is in LD with putative genetic variant(s).

We have shown previously that the neuropathology of ET, in the majority of cases, is characterized by a marked increase in torpedoes (axonal swelling of Purkinje cells) and Purkinje cell loss compared with their occurrence in normal aging. ${ }^{7,22,23}$ The normal function of LINGO1 in axon regeneration, central nervous system myelination and regulation of neuronal survival, together with the neuropathological features of ET, suggests a possible role for LINGO1 in the pathophysiology of this disease. ${ }^{14}$ In animal CNS disease models that have targeted LINGO1 inhibition, neuron and oligodendrocyte survival, axon regeneration, oligodendrocyte differentiation, remyelination and improved functional recovery were promoted. ${ }^{24}$ Although the mechanism by which rs 9652490 and/or additional genetic variants in LINGO1 lead to disease is unknown, functional studies of the 'normal' LINGO1 gene suggest that disease-associated risk factors may

Table 4 Summary of allelic association

\begin{tabular}{|c|c|c|c|c|c|c|c|c|c|c|}
\hline SNPa & Studies ${ }^{\mathrm{b}}$ & Allele 1 & Allele 2 & Freq Allele 1 & Freq SE Allele 1 & Min Freq & Max Freq & Weight $^{\mathrm{c}}$ & $Z$ score $^{d}$ & P-value \\
\hline \multicolumn{11}{|c|}{ (a) Three replication studies combined } \\
\hline rs3743481 & 1,4 & $\mathrm{~T}$ & $\mathrm{C}$ & 0.408 & 0.024 & 0.395 & 0.453 & 2376 & 0.75 & 0.4534 \\
\hline rs9652490 & $1,3,4$ & G & A & 0.242 & 0.014 & 0.231 & 0.263 & 3305 & 3.39 & 0.0007 \\
\hline rs3144 & 1,4 & $\mathrm{C}$ & $\mathrm{T}$ & 0.312 & 0.030 & 0.255 & 0.328 & 2374 & 2.25 & 0.0242 \\
\hline rs8028808 & 1,4 & $A$ & $\mathrm{G}$ & 0.168 & 0.012 & 0.144 & 0.174 & 2382 & 3.18 & 0.0015 \\
\hline \multicolumn{11}{|c|}{ (b) All four studies combined } \\
\hline rs3743481 & 1,4 & $\mathrm{~T}$ & $\mathrm{C}$ & 0.408 & 0.024 & 0.395 & 0.453 & 2376 & 0.75 & 0.4534 \\
\hline rs9652490 & $1,2,3,4$ & G & A & 0.310 & 0.034 & 0.231 & 0.329 & 19854 & 6.74 & $1.55 \mathrm{E}-11$ \\
\hline rs3144 & 1,4 & C & $\mathrm{T}$ & 0.312 & 0.030 & 0.255 & 0.328 & 2374 & 2.25 & 0.02421 \\
\hline rs8028808 & 1,4 & A & G & 0.168 & 0.012 & 0.144 & 0.174 & 2382 & 3.18 & 0.00148 \\
\hline
\end{tabular}

Only rs9652490 was genotyped in all studies. For each of the remaining SNPs, studies that had the genotype are indicated:

aln this study.

bStefansson et al. ${ }^{14}$

cTan et al. 16

dVilariño-Güell et al. ${ }^{15}$ When alleles were called differently, alleles from this study were used. Weight represents the total number of subjects genotyped. The direction of the $Z$ score represents allelic association with allele 1. 
lead to 'a gain-of-function' or 'overexpression' of LINGO1. Future studies, including 'deep' sequencing and gene expression studies, will be needed to clarify the genetic contribution of rs9652490 and additional sequence variants in LINGO1 to disease risk and functional studies to determine the disease mechanism.

\section{CONFLICT OF INTEREST}

The authors declare no conflict of interest.

\section{ACKNOWLEDGEMENTS}

This study was supported by The National Institutes of Health (R01 NS42859 to EDL, R01 NS39422 to EDL and P30 ES009089 to EDL), the Parkinson's Disease Foundation (New York, NY) to EDL and LNC and the Arlene Bronstein Essential Tremor Research Fund (Columbia University) to EDL. Technical support was provided by Prashanthi Maramreddy (Columbia University).

1 Benito-Leon J, Louis ED: Essential tremor: emerging views of a common disorder. Nat Clin Pract Neurol 2006; 2: 666-678.

2 Louis ED, Thawani SP, Andrews HF: Prevalence of essential tremor in a multiethnic, community-based study in northern Manhattan, New York, NY. Neuroepidemiology 2009; 32: 208-214.

3 Gulcher JR, Jonsson P, Kong A et al: Mapping of a familial essential tremor gene, FET1, to chromosome 3q13. Nat Genet 1997; 17: 84-87.

4 Higgins JJ, Pho LT, Nee LE: A gene (ETM) for essential tremor maps to chromosome 2p22-p25. Mov Disord 1997; 12: 859-864.

5 Shatunov A, Sambuughin N, Jankovic J et al: Genomewide scans in North American families reveal genetic linkage of essential tremor to a region on chromosome $6 \mathrm{p} 23$. Brain 2006; 129: 2318-2331.

6 Louis ED, Levy G, Mejia-Santana $\mathrm{H}$ et al: Risk of action tremor in relatives of tremordominant and postural instability gait disorder PD. Neurology 2003; 61: 931-936.

7 Louis ED, Faust PL, Vonsattel JP et al: Neuropathological changes in essential tremor: 33 cases compared with 21 controls. Brain 2007; 130: 3297-3307.
8 Benito-Leon J, Louis ED, Bermejo-Pareja F: Risk of incident Parkinson's disease and parkinsonism in essential tremor: a population based study. J Neurol Neurosurg Psychiatry 2009; 80: 423-425.

9 Deng H, Le W, Davidson AL, Xie W, Jankovic J: The LRRK2 I2012T, G2019S and I2020T mutations are not common in patients with essential tremor. Neurosci Lett 2006; 407: 97-100.

10 Deng H, Le W, Huang M, Xie W, Pan T, Jankovic J: Genetic analysis of LRRK2 P755L variant in Caucasian patients with Parkinson's disease. Neurosci Lett 2007; 419: 104-107.

11 Tan EK, Lee J, Lim HQ, Yuen Y, Zhao Y: Essential tremor and the common LRRK2 G2385R variant. Parkinsonism Relat Disord 2008; 14: 569-571.

12 Vitale C, Ciotti P, Gulli R et al: Common mutations in the LRRK2 exon 41 are not responsible for essential tremor in Italian patients. Parkinsonism Relat Disord 2009; 15: 162-163.

13 Clark LN, Kisselev S, Park N et al: Mutations in the Parkinson's disease genes, Leucine rich repeat Kinase 2 (LRRK2) and Glucocerebrosidase (GBA), are not associated with essential tremor. Parkinsonism Relat Disord 2010; 16: 132-135.

14 Stefansson H, Steinberg S, Petursson $\mathrm{H}$ et al: Variant in the sequence of the LINGO1 gene confers risk of essential tremor. Nat Genet 2009; 41: 277-279.

15 Vilariño-Güell C, Ross OA, Wider C et al: LINGO1 rs9652490 is associated with essential tremor and Parkinson disease. Parkinsonism Relat Disord 2010; 16: 109-111.

16 Tan EK, Teo YY, Prakash KM et al: LINGO1 variant increases risk of familial essential tremor. Neurology 2009; 73: 1161-1162.

17 Louis ED, Schonberger RB, Parides M, Ford B, Barnes LF: Test-retest reliability of patient information on age of onset in essential tremor. Mov Disord 2000; 15: 738-741.

18 Louis ED, Dogu O: Does age of onset in essential tremor have a bimodal distribution? Data from a tertiary referral setting and a population-based study. Neuroepidemiology 2007; 29: 208-212.

19 Louis ED, Jiang W, Pellegrino KM et al: Elevated blood harmane (1-methyl-9H-pyrido[3,4b]indole) concentrations in essential tremor. Neurotoxicology 2008; 29: 294-300.

20 Barrett JC, Fry B, Maller J, Daly MJ: Haploview: analysis and visualization of LD and haplotype maps. Bioinformatics 2005; 21: 263-265.

21 Schaid DJ, Rowland CM, Tines DE, Jacobson RM, Poland GA: Score tests for association between traits and haplotypes when linkage phase is ambiguous. $A m \mathrm{~J}$ Hum Genet 2002; 70: 425-434.

22 Louis ED, Faust PL, Vonsattel JP, Erickson-Davis C: Purkinje cell axonal torpedoes are unrelated to advanced aging and likely reflect cerebellar injury. Acta Neuropathol 2009; 117: 719-721.

23 Louis ED, Yi H, Erickson-Davis C, Vonsattel JP, Faust PL: Structural study of Purkinje cell axonal torpedoes in essential tremor. Neurosci Lett 2009; 450: 287-291.

$24 \mathrm{Mi} \mathrm{S}$, Hu B, Hahm K et al: LINGO-1 antagonist promotes spinal cord remyelination and axonal integrity in MOG-induced experimental autoimmune encephalomyelitis. Nat Med 2007; 13: 1228-1233.

Supplementary Information accompanies the paper on European Journal of Human Genetics website (http://www.nature.com/ejhg) 